\title{
Effect of rapamycin on early stage apoptosis of neutrophils in Sprague-Dawley rats with acute lung injury
}

\author{
LIWEI LI* ${ }^{*}$, CHANGTAI ZHU*, YE YUAN and ZHIQIANG LI* \\ Department of Transfusion, Shanghai Jiao Tong University Affiliated Sixth People's Hospital, Shanghai 200233, P.R. China
}

Received December 13, 2016; Accepted June 9, 2017

DOI: $10.3892 /$ br.2017.936

\begin{abstract}
Transfusion-related acute lung injury (TRALI) is a serious complication characterized by the acute onset of non-cardiogenic pulmonary edema following transfusion of blood products. It is not known whether rapamycin has an effect on TRALI that is caused by blood transfusion. The aim of the present study was to determine the effect of rapamycin on early stage apoptosis of neutrophils in Sprague-Dawley rats with acute lung injury. Animal models of TRALI and acute pancreatitis-associated lung injury (APALI) were prepared using Sprague-Dawley rats and histopathological examination of lung tissues was used to validate acute lung injury models. Peripheral blood neutrophils were isolated and early stage apoptosis of neutrophils was detected by flow cytometry. The animal models of TRALI and APALI were constructed successfully. Early stage apoptosis of neutrophils increased in the TRALI group and decreased in the APALI group $(\mathrm{P}<0.05)$, while the apoptosis rates in rapamycin intervention TRALI and APALI groups were not significantly different compared to the rate in the control group $(\mathrm{P}>0.05)$. In conclusion, early stage apoptosis of neutrophils in TRALI was different from that in APALI, and rapamycin had a limited protective effect on TRALI and APALI in Sprague-Dawley rats.
\end{abstract}

\section{Introduction}

Transfusion-related acute lung injury (TRALI) is a serious complication characterized by the acute onset of non-cardiogenic pulmonary edema following transfusion of blood products. Although its incidence decreased with modified transfusion practices, TRALI was the leading cause of transfusion-related fatalities in the United States from 2007

Correspondence to: Dr Zhiqiang Li, Department of Transfusion, Shanghai Jiao Tong University Affiliated Sixth People's Hospital, 600 Yishan Road, Shanghai 200233, P.R. China

E-mail: liuyuanlzq@163.com

*Contributed equally

Key words: acute lung injury, transfusion, pancreatitis, neutrophil apoptosis through to 2011 (1). Acute pancreatitis-associated lung injury (APALI) is the most common and a severe complication in patients with multisystem organ failure. Previous studies indicated that neutrophils serve a critical role in inflammatory reactions in acute lung injury $(2,3)$.

Rapamycin is a new macrolide immunosuppressant. Rapamycin exerts an immunosuppressive effect by blocking signal transduction of various cytokine receptors, thereby preventing T lymphocytes and other cells from going from the G1 phase to the $\mathrm{S}$ phase of the cell cycle. A recent report has also indicated that rapamycin can inhibit the inflammatory reaction induced by nuclear factor $(\mathrm{NF})-\kappa \mathrm{B}$ activation and neutrophils $(2,3)$. Rapamycin also reportedly exacerbates the severity of acute lung injury induced by endotoxin and causes cell apoptosis (4).

It is unknown whether rapamycin has an effect on TRALI caused by blood transfusion. Therefore, the authors constructed Sprague-Dawley rat models of TRALI and APALI, and observed the effect of rapamycin on the pathological process of neutrophil apoptosis in the early stage of acute lung injury induced by blood transfusion, in order to obtain experimental evidence for future clinical treatment feasibility.

\section{Materials and methods}

Ethics statement. The current study was approved by the Shanghai Jiao Tong University Affiliated Sixth People's Hospital of Medicine Ethics Committee (Shanghai, China).

Animals. A total of 60 male Sprague-Dawley rats aged 6-7 weeks (weight, $250 \mathrm{~g}$ ) were purchased from Shanghai SIPPR/BK Experimental Animals, Inc. (Shanghai, China). The rats were reared to be specific pathogen free, and standard feed, drinking water, bedding, and all other items were aseptically processed. The rats were maintained with a $12 \mathrm{~h}: 12 \mathrm{~h}$ light cycle.

Instruments and reagents. The main instruments included a low-temperature high-speed centrifuge (Allegra X-22R; Beckman Coulter, Inc., Brea, CA, USA), and flow cytometer (Navios; Beckman Coulter, Inc.). The main regents were as follows: Lipopolysaccharide (Sigma-Aldrich; Merck KGaA, Darmstadt, Germany), L-arginine (Amresco, Inc., Framingham, MA, USA), kits for isolation of rat peripheral blood neutrophils (cat. no. LZS1091), red blood cell lysis buffer (cat. no. NH4CL2009), cell dilution (cat. no. 2010C1119), cell 
washing liquid (cat. no. 2010X1118) and Annexin V-fluorescein isothiocyanate (FITC)/propidium iodide (PI) cell apoptosis detection kit (cat. no. 40302ES20; Shanghai Yi Sheng Biotechnology Co., Ltd., Shanghai, China).

Plasma preparation. AB type human whole blood was randomly selected, and $2 \mathrm{ml}$ blood samples were set aside. Following 21 days of storage, plasma was obtained by centrifugation at $1,200 \mathrm{x} g$ and then preserved at $-80^{\circ} \mathrm{C}$. Before infusion, plasma was incubated at $56^{\circ} \mathrm{C}$ for $30 \mathrm{~min}$.

Preparation of animal models. A total of 60 Sprague-Dawley rats were randomly divided into 6 groups (10 rats in each group): A normal control group, a TRALI experimental group, an APALI experimental group, a rapamycin intervention control group, a rapamycin intervention TRALI experimental group, and a rapamycin intervention APALI experimental group. In order to construct acute lung injury models, the rats were treated as follows. The TRALI group rats received intraperitoneal injection of LPS $(2 \mathrm{mg} / \mathrm{kg})$. At $1 \mathrm{~h}$, the rats were anesthetized by intraperitoneal injection of pentobarbital anesthesia (40 mg/ $\mathrm{kg}$ ); femoral vessels were isolated and infused with $50 \%$ heparin-saline mixture. Subsequently, $1 \mathrm{ml}$ blood [ $10 \%$ of the capacity of whole blood was removed within 5-10 min, and the equivalent AB human plasma was transfused (plasma infusion rate less than $4 \mathrm{ml} / \mathrm{h}$ )] (5). The APALI group rats received 3 intraperitoneal injections of $9 \%$ L-arginine solution (dilute with physiological saline) $(1 \mathrm{~g} / \mathrm{kg})$ at $1 \mathrm{~h}$ intervals (5). The rapamycin intervention experimental TRALI and APALI group rats underwent intragastric administration of rapamycin (10 mg/kg, once a day for 3 days), and other experimental methods were as in the above protocols. Finally, the rapamycin intervention control group rats in the TRALI and APALI control groups were intraperitoneally injected with rapamycin in saline $(2 \mathrm{mg})$. Under anesthesia, $1 \mathrm{ml}$ blood was removed from the rats and $1 \mathrm{ml}$ of normal saline was transfused. The ensuing experimental operation for the control group was the same as previously described for the TRALI and APALI group.

Histopathological examination of lung tissues. The rats were killed by right ventricular puncture, and lung tissue specimens $(1 \mathrm{x} 1 \mathrm{~cm})$ were collected. Hematoxylin and eosin staining was used for histopathological examination. There were 60 images randomly selected for analysis.

Isolation of neutrophils in peripheral blood. As per the kit instructions: $1 \mathrm{ml}$ fresh anticoagulated blood was added onto the surface of a $1 \mathrm{ml}$ isolation liquid and centrifuged at $400-500 \mathrm{x} g$ for $25 \mathrm{~min}$. The centrifuge tube will have been divided into 4 layers; the liquid layers rich in neutrophils and red blood cells are drawn off. Following washing, red blood cell lysis solution is used to completely destroy the red blood cells in the rat peripheral blood. The supernatant is discarded by centrifuging at $\sim 400-500 \mathrm{x}$ g for $5 \mathrm{~min}$ and the precipitate is washed twice with a cell washing solution to obtain useful neutrophils.

Quantitative detection of early stage neutrophil apoptosis. The Annexin V and PI double-labeling method was used
Table I. Comparisons of the early apoptosis rate of peripheral blood neutrophils in the rats.

\begin{tabular}{lc}
\hline Group & $\begin{array}{c}\text { Neutrophil apoptosis rate } \\
(\mathrm{n}=10)\end{array}$ \\
\hline Rapamycin intervention TRALI & $18.84 \pm 1.94^{\mathrm{a}}$ \\
TRALI experimental & $18.06 \pm 1.66^{\mathrm{acc}}$ \\
Rapamycin intervention APALI & $7.95 \pm 1.92^{\mathrm{b}-\mathrm{d}}$ \\
APALI experimental & $10.00 \pm 3.12^{\mathrm{b}}$ \\
Rapamycin intervention control & $11.32 \pm 4.23$ \\
Normal control & $13.35 \pm 3.68$ \\
\hline
\end{tabular}

TRALI, transfusion related acute lung injury; APALI, acute pancreatitis associated lung injury. ${ }^{\mathrm{a}} \mathrm{P}<0.01 \mathrm{vs}$. normal control; ${ }^{\mathrm{b}} \mathrm{P}<0.05$ vs. normal control; ${ }^{\mathrm{c}} \mathrm{P}<0.05$ vs. APALI group; ${ }^{\mathrm{d}} \mathrm{P}<0.05$ vs. rapamycin intervention TRALI group.

according to manufacturer's instructions. The separation and purification of neutrophils is suspended in $100 \mu \mathrm{l}$ buffer, $5 \mu \mathrm{l}$ Annexin V-FLUOS, and $5 \mu \mathrm{l} \mathrm{PI}$ at room temperature, with dark incubation for $10 \mathrm{~min} ; 400 \mu \mathrm{l}$ incubation buffer solution from the Annexin V-fluorescein isothiocyanate (FITC)/propidium iodide (PI) cell apoptosis detection kit (cat. no. 40302ES20; Shanghai Yi Sheng Biotechnology Co., Ltd.)] was added to terminate the reaction. A total of 10,000 cells are detected by dual-channel flow cytometry.

Statistical analysis. The data were analyzed using SPSS software (version, 17.0; SPSS Inc., Chicago, IL, USA). Continuous variables were described using mean \pm standard deviation and the comparison between groups was performed with a t-test or correlation analysis. $\mathrm{P}<0.05$ was considered to indicate a statistically significant difference.

\section{Results}

Histopathological examination of lung tissues. Lung tissue pathological changes in the TRALI and APALI groups included significantly widened alveolar intervals, damage and fracture of alveolar walls and capillaries, congestion, erythrocyte effusion in organization gaps, a large number of inflammatory cells in the alveolar spaces, and inflammatory neutrophil infiltration in interstitial spaces (Figs. 1 and 2). However, the control group reported no significant changes (Fig. 3). Therefore, the rat models were successfully developed.

Changes of early neutrophil apoptosis in peripheral blood in rats with TRALI. Compared with the normal control group, the early peripheral blood neutrophil apoptosis rate in the TRALI experimental group increased $(\mathrm{P}<0.01$; Fig. 4$)$.

There was no difference in the early peripheral blood neutrophil apoptosis rate between the rapamycin intervention TRALI group and the TRALI control group, while the early neutrophil apoptosis rate in the TRALI rapamycin intervention group was higher than that in the control group $(\mathrm{P}<0.01$; Figs. 5 and 6). 


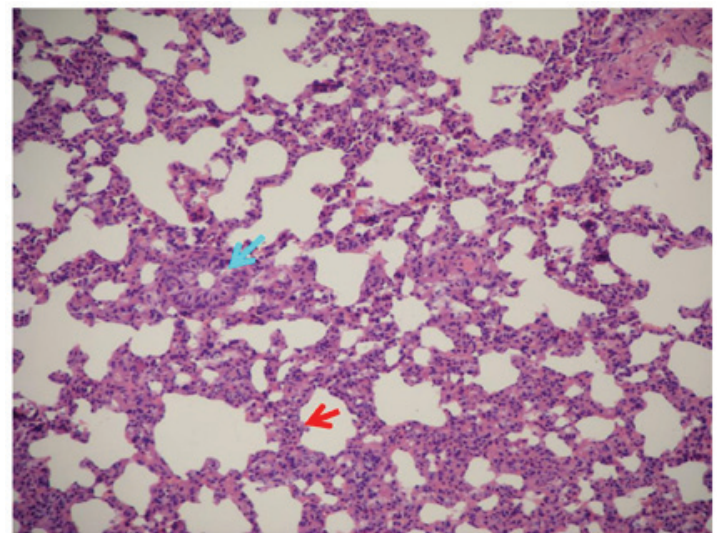

Figure 1. The histopathological examination of lung tissues in acute lung injury rats (magnification, x10). Blue arrow, thickening of the bronchi; red arrow, thickening of alveolar interval.

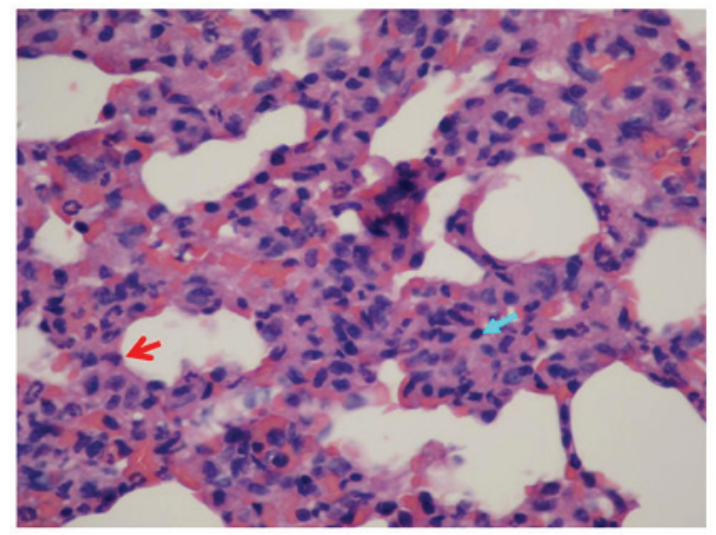

Figure 2. The histopathological examination in lung tissues in rats of transfusion-related acute lung injury group (magnification, $x 40$ ). Blue arrow, neutrophil infiltration; red arrow, thickening of alveolar interval.

The early apoptosis rate in the TRALI rapamycin intervention experimental group was significantly higher than that in the control group $(\mathrm{P}<0.01$; Table I).

Changes of early neutrophil apoptosis in peripheral blood in rats with APALI. The early apoptosis rates in both the APALI experimental group and rapamycin intervention APALI group were lower than those in the normal control group $(\mathrm{P}<0.05$; Fig. 4). There was no difference in the early apoptosis rates between the rapamycin intervention APALI group and the APALI experimental group $(\mathrm{P}>0.05)$.

The early apoptosis rate in the rapamycin intervention APALI group was lower than that in the APALI experimental group $(\mathrm{P}<0.05)$. There was no difference in the early apoptosis rates of neutrophils between the rapamycin intervention APALI group and the control group ( $\mathrm{P}>0.05$; Table I).

Comparison of the early apoptosis rates of peripheral blood neutrophils in the TRALI experimental group and APALI experimental group. Compared with that in the APALI group, the early apoptosis rate of peripheral blood neutrophils significantly increased in the TRALI group $(\mathrm{P}<0.05)$. Following rapamycin intervention, the early apoptosis rate of peripheral blood neutrophils in the TRALI experimental group was

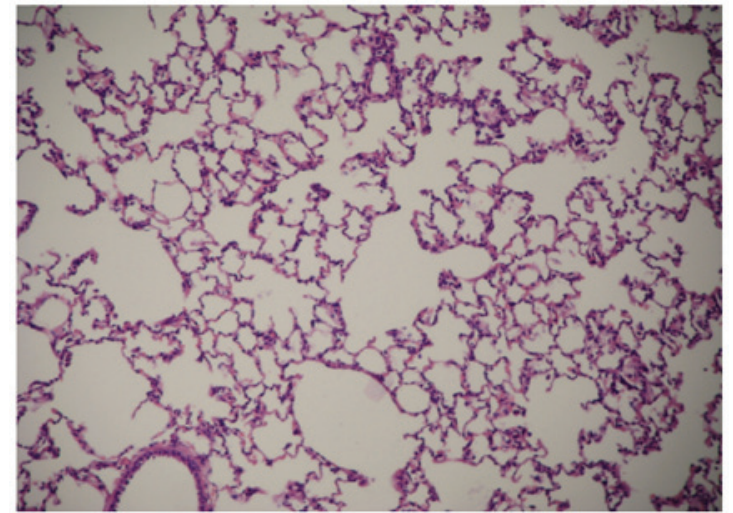

Figure 3. The histopathological examination of lung tissues in normal control rats (magnification, x10).

significantly higher than that in the APALI experimental group $(\mathrm{P}<0.05$; Table I).

Another study (6) that was performed by the authors indicated that $10 \mathrm{mg} / \mathrm{kg}$ rapamycin can significantly reduce the expression of p-p70s6k, which confirmed mTOR activation was inhibited in the experimental dose of rapamycin in the current study.

\section{Discussion}

A specific inhibitor of the mammalian target of rapamycin (mTOR) is a commonly used pharmacological tool for the study of mTOR biology (7). As a key controller of cell growth and survival, the mTOR can physically interact with STAT1 and suppress the induction of STAT1-dependent apoptosis genes (3). The mTOR can also modify gene transcription by directly regulating transcription factors. There are two functionally distinct mammalian targets of rapamycin complexes (mTORCs), mTORC1 and mTORC2, with mTORC1 serving a central role in cell growth and cellular responses to metabolic stress. mTORC1 participates in the regulation of transcriptional as well as translational events for genes involved in biosynthetic and metabolism pathways (6-9). In contrast, mTORC2 controls the organization of the actin cytoskeleton and is rapamycin insensitive (10).

Acute lung injury (ALI) is characterized by lung inflammation and diffuses infiltration of neutrophils into the alveolar space. The inhibition of neutrophil apoptosis has been implicated in the pathogenesis of ALI. It has been proposed that activated neutrophils in the lungs have an unusually prolonged half-life of $8 \mathrm{~h}$ due to delayed phagocytosis (or apoptosis) by macrophages (11). In a septic patient, this delay appears to be related to the severity of sepsis since a progressive decrease in neutrophil apoptosis has been associated with the increased severity of sepsis (12). The inhibition of neutrophil apoptosis may contribute to the development of ALI (13). It was reported that lung inflammation could be ameliorated by enhancing the apoptosis of neutrophils (14). Depletion of neutrophils substantially mitigated the extent of lung injury, indicating a pathomechanistic role for neutrophils in chest trauma-induced septic ALI (15). Inflammasomes have been reported to be involved in ALI in previous studies (16,17). Histone deacetylase 9 inhibited autophagy in primary brain microvessel endothelial 

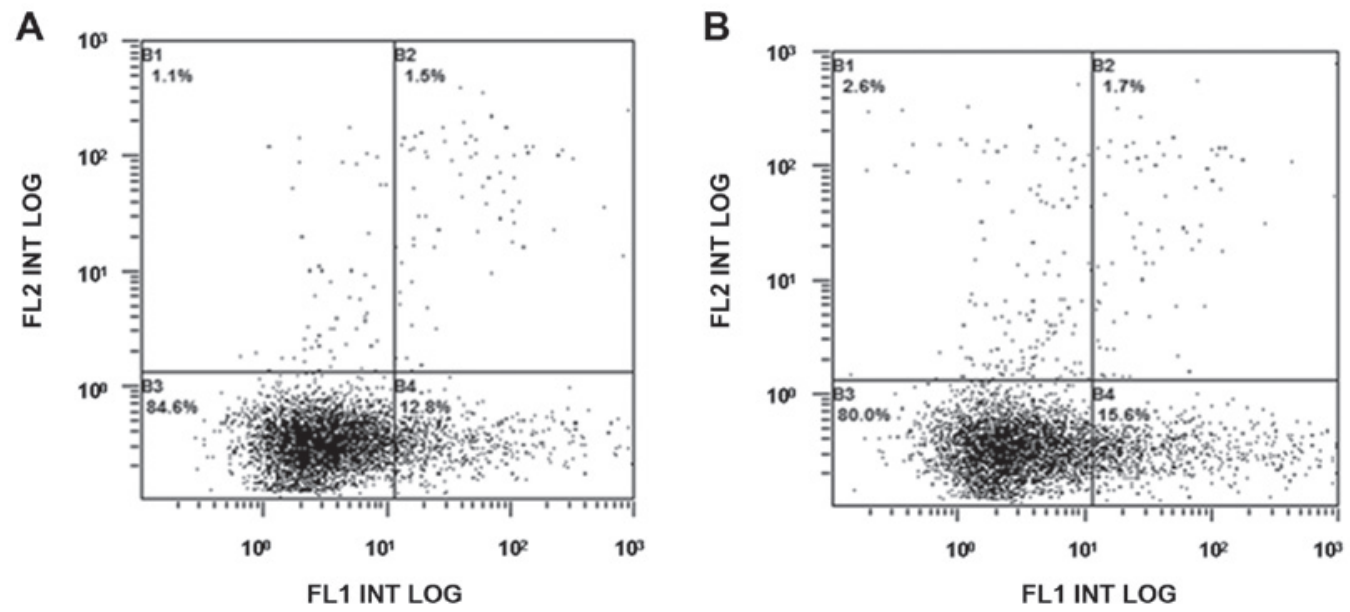

Figure 4. Quantitative detection of neutrophil early apoptosis in normal control and TRALI experimental groups by flow cytometry. (A) Normal control group; (B) TRALI experimental group. TRALI, transfusion-related acute lung injury.
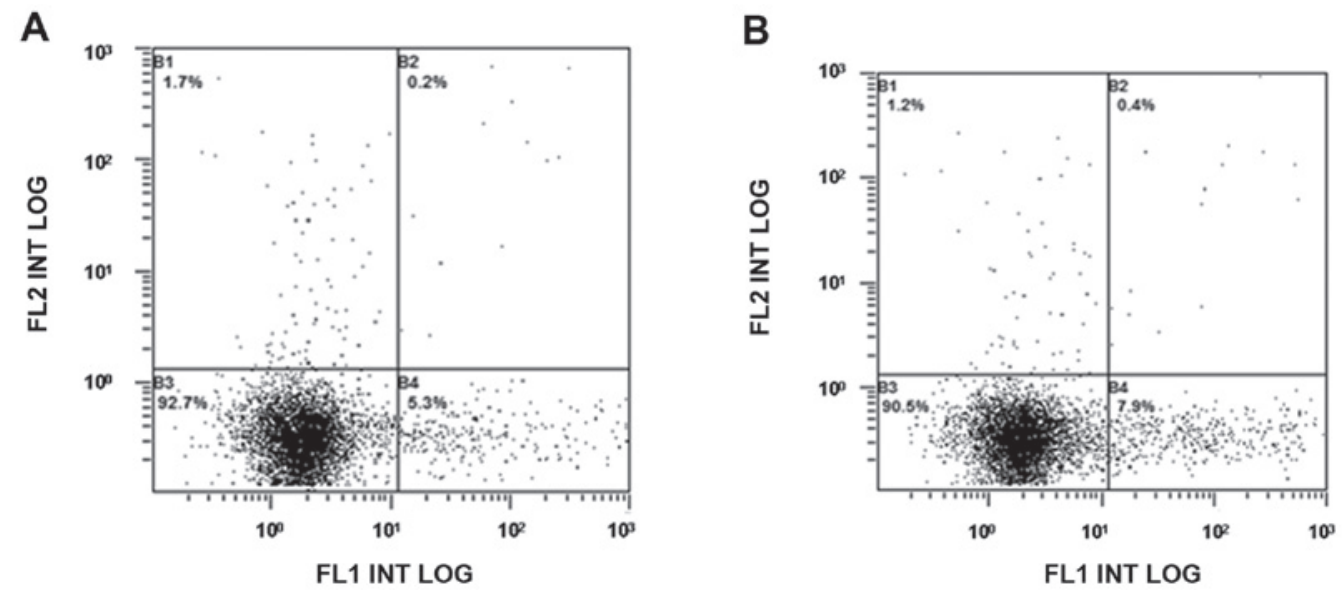

Figure 5. Quantitative detection of neutrophil early apoptosis in APALI experimental group and rapamycin intervention control group. (A) APALI experimental group; (B) rapamycin intervention control group. APALI, acute pancreatitis-associated lung injury.
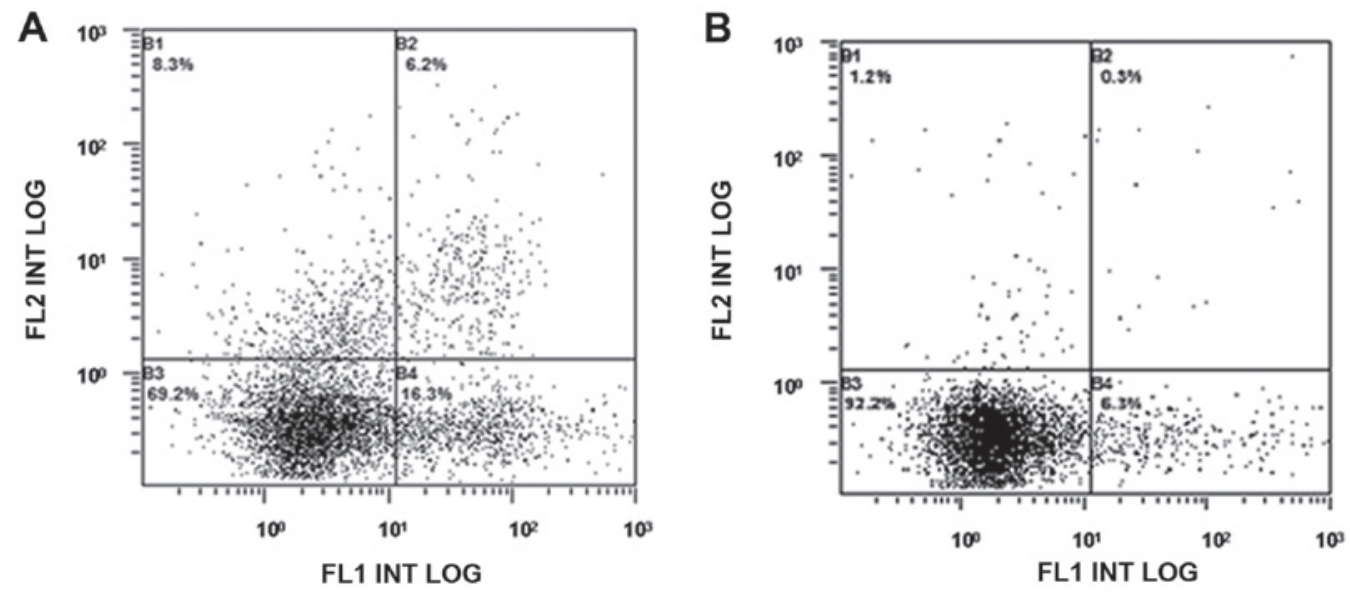

Figure 6. Quantitative detection of neutrophil early apoptosis in rapamycin intervention TRALI and rapamycin intervention APALI experimental groups. (A) Rapamycin intervention TRALI experimental group; (B) rapamycin intervention APALI experimental group. TRALI, transfusion-related acute lung injury; APALI, acute pancreatitis-associated lung injury.

cells that exacerbates brain ischemia injury (16). However, rapamycin can induce protective autophagy in vascular endothelial cells exposed to oxygen-glucose deprivation (17).
Rapamycin is approved by the U.S. Food and Drug Administration for immunosuppression. It has been reported that rapamycin decreased the severity of lung injury after 
intratracheal LPS or PAM administration, as determined by diminished neutrophil accumulation in the lungs and reduced interstitial pulmonary edema (18). The downregulation of autophagy may lead to systemic inflammation and ALI following sepsis. The direct or indirect modification of autophagy using rapamycin, respectively, resulted in improved survival. Enhancing or restoring autophagy early after sepsis seems to be a potential strategy for the treatment of sepsis-induced ALI (19). However, rapamycin can reduce paraquat-induced ALI through inhibition of NF- $\mathrm{kB}$ activation, as well as increased apoptosis of leukocytes and neutrophils (20).

The current study demonstrated that the early stage apoptosis of neutrophils in Sprague-Dawley rats with APALI was decreased, indicating that peripheral neutrophil apoptosis was delayed, which occurs frequently in patients with burns, infection and multi-organ failure (21), and further increases the severity of lung injury (22).

However, early stage apoptosis of neutrophils in the rapamycin intervention group did not change significantly compared to that in the control group, and lung injury did not improve following rapamycin intervention, suggesting that rapamycin did not change the early stage apoptosis rate of neutrophils. In addition, rapamycin did not affect early stage apoptosis of neutrophils in the APALI group according to our results. Therefore, the authors believe that rapamycin had limited protective effects on APALI, but did not relieve APALI.

The early apoptosis rate of neutrophils in the TRALI group was increased compared to that in the normal control group in the current study, but rapamycin had little effect on neutrophil apoptosis in TRALI. Compared with the APALI group, with or without rapamycin intervention, delayed neutrophil apoptosis did not occur in TRALI. The activation and inactivation of mTOR depend on the different stimuli and different types of cells and the differential regulations under the influence of these factors lead to the different effects of rapamycin on mTOR (6). The authors believe that early stage apoptosis of neutrophils in TRALI was different from that in APALI, and rapamycin had a limited protective effect on TRALI and APALI in Sprague Dawley rats. With future research, better treatments for acute lung injury with more targeted effects may be developed.

\section{Acknowledgements}

The current study was funded by the Fourth Round (2015-2017) Shanghai Public Health Priority Construction Project, 'Transfusion Medicine' (grant no.15GWZK0501), Shanghai Science and Technology Commission Research Project Funding (grant no. 13140902903), and Shanghai Sixth People's Hospital Consortium Subject (2015; grant no. 1324).

\section{References}

1. Menis M, Anderson SA, Forshee RA, McKean S, Johnson C, Warnock R, Gondalia R, Mintz PD, Holness L, Worrall CM, et al: Transfusion-related acute lung injury and potential risk factors among the inpatient US elderly as recorded in Medicare claims data, during 2007 through 2011. Transfusion 54: 2182-2193, 2014

2. Abraham E: Neutrophils and acute lung injury. Crit Care Med 31: S195-S199, 2003.
3. Fielhaber JA, Carroll SF, Dydensborg AB, Shourian M, Triantafillopoulos A, Harel S, Hussain SN, Bouchard M, Qureshi ST and Kristof AS: Inhibition of mammalian target of rapamycin augments lipopolysaccharide-induced lung injury and apoptosis. J Immunol 188: 4535-4542, 2012.

4. Mossman JA, Biancani LM, Zhu CT and Rand DM: Mitonuclear Epistasis for Development Time and Its Modification by Diet in Drosophila. Genetics 203: 463-484, 2016.

5. Sharif R, Dawra R, Wasiluk K, Phillips P, Dudeja V, Kurt-Jones E, Finberg R and Saluja A: Impact of toll-like receptor 4 on the severity of acute pancreatitis and pancreatitis-associated lung injury in mice. Gut 58: 813-819, 2009.

6. Yuan YE, Liwei L and Zhiqiang L: Effects of rapamycin on expressions of $\mathrm{m}$ TOR down-stream proteins $\mathrm{p} 70 \mathrm{~s} 6 \mathrm{k} / \mathrm{p}-\mathrm{p} 70 \mathrm{~s} 6 \mathrm{k}$ and pulmonary histopathological changes in transfusion-related acute lung injury rat model. Chin J Blood Transfus 29: 251-254, 2016.

7. Hay N and Sonenberg N: Upstream and downstream of mTOR. Genes Dev 18: 1926-1945, 2004.

8. Tee AR and Blenis J: mTOR, translational control and human disease. Semin Cell Dev Biol 16: 29-37, 2005.

9. Peng T, Golub TR and Sabatini DM: The immunosuppressant rapamycin mimics a starvation-like signal distinct from amino acid and glucose deprivation. Mol Cell Biol 22: 5575-5584, 2002.

10. Jacinto E, Loewith R, Schmidt A, Lin S, Rüegg MA, Hall A and Hall MN: Mammalian TOR complex 2 controls the actin cytoskeleton and is rapamycin insensitive. Nat Cell Biol 6: 1122-1128, 2004.

11. Cheretakis C, Dror Y and Glogauer M: A noninvasive oral rinse assay to monitor engraftment, neutrophil tissue delivery and susceptibility to infection following HSCT in pediatric patients. Bone Marrow Transplant 36: 227-232, 2005.

12. Fialkow L, Fochesatto Filho L, Bozzetti MC, Milani AR, Rodrigues Filho EM, Ladniuk RM, Pierozan P, de Moura RM, Prolla JC, Vachon E, et al: Neutrophil apoptosis: A marker of disease severity in sepsis and sepsis-induced acute respiratory distress syndrome. Crit Care 10: R155, 2006.

13. Lin WC, Lin CF, Chen CL, Chen CW and Lin YS: Inhibition of neutrophil apoptosis via sphingolipid signaling in acute lung injury. J Pharmacol Exp Ther 339: 45-53, 2011.

14. Perl M, Hohmann C, Denk S, Kellermann P, Lu D, Braumüller S, Bachem MG, Thomas J, Knöferl MW, Ayala A, et al: Role of activated neutrophils in chest trauma-induced septic acute lung injury. Shock 38: 98-106, 2012.

15. Li X, Li C, Liang W, Bi Y, Chen M and Dong S: Protectin D1 promotes resolution of inflammation in a murine model of lipopolysaccharide-induced acute lung injury via enhancing neutrophil apoptosis. Chin Med J (Engl) 127: 810-814, 2014.

16. Shi W, Wei X, Wang Z, Han H, Fu Y, Liu J, Zhang Y, Guo J, Dong C, Zhou D, et al: HDAC9 exacerbates endothelial injury in cerebral ischaemia/reperfusion injury. J Cell Mol Med 20: 1139-1149, 2016.

17. Urbanek T, Kuczmik W, Basta-Kaim A and Gabryel B: Rapamycin induces of protective autophagy in vascular endothelial cells exposed to oxygen-glucose deprivation. Brain Res 1553: 1-11, 2014.

18. Lorne E, Zhao X, Zmijewski JW, Liu G, Park YJ, Tsuruta Y and Abraham E: Participation of mammalian target of rapamycin complex 1 in Toll-like receptor 2- and 4-induced neutrophil activation and acute lung injury. Am J Respir Cell Mol Biol 41: 237-245, 2009.

19. Yen YT, Yang HR, Lo HC, Hsieh YC, Tsai SC, Hong CW and Hsieh $\mathrm{CH}$ : Enhancing autophagy with activated protein $\mathrm{C}$ and rapamycin protects against sepsis-induced acute lung injury. Surgery 153: 689-698, 2013.

20. Chen D, Ma T, Liu XW, Yang C and Liu Z: Rapamycin reverses paraquat-induced acute lung injury in a rat model through inhibition of NFxB activation. Int J Clin Exp Pathol 8: 4627-4638, 2015.

21. Taneja R, Parodo J, Jia SH, Kapus A, Rotstein OD and Marshall JC: Delayed neutrophil apoptosis in sepsis is associated with maintenance of mitochondrial transmembrane potential and reduced caspase-9 activity. Crit Care Med 32: 1460-1469, 2004.

22. O'Neill S, O'Neill AJ, Conroy E, Brady HR, Fitzpatrick JM and Watson RW: Altered caspase expression results in delayed neutrophil apoptosis in acute pancreatitis. J Leukoc Biol 68: $15-20,2000$. 